\title{
Evaluation de la stabilité et la maturité des composts obtenus par biodégradation aérobie d'un mélange de déchets ménagers et de déchets de poulets
}

\author{
Mina Aylaj et El Kbir Lhadi \\ Laboratoire de l'eau et de l'environnement, Faculté des Sciences - BP 20, 24000 El Jadida (Maroc)
}

Pour toute correspondance : mina_aylaj@yahoo.fr

\begin{abstract}
Résumé
La nature des déchets ménagers (DM) et des déchets de poulets (DP) de la ville d'El Jadida (Maroc) et sa vocation agricole ont nécessité une réflexion sur l'étude de la faisabilité du compostage comme voie de traitement de déchets solides. Deux proportions ont été utilisées: proportion de 3/2 (compost $\mathrm{Cl}$ ) et de 2/3 (compost C2), rapport masse frâche DM/masse fraîche DP). Ces deux mélanges sont introduits dans deux réacteurs pour subir un compostage aérobie actif pendant 15 jours : c'est la phase de compostage. Les produits obtenus ont subi ensuite une dégradation lente dans des sacs perforés pendant 23I jours : c'est la phase de maturation. L'évaluation de la maturité des composts obtenus se fait par un test de phytotoxicité. Les résultats obtenus montrent que l'âge du compost affecte significativement le pourcentage et l'indice de germination (IG). Le compost est considéré stabilisé et mûr dès que IG dépasse $60 \%$. Cette valeur est atteinte après 105 jours pour les deux composts.

Le rapport $\mathrm{C} / \mathrm{N}$ diminue au cours de la phase de maturation et atteint 6,3 et 6 respectivement pour $\mathrm{Cl}$ et $\mathrm{C} 2$ à 246 jours de compostage. La quantité d'azote ammoniacal décroît progressivement alors que la quantité de nitrate augmente. Les composts obtenus semblent très similaires et de bonne qualité, suggérant que le compostage peut être une bonne voie pour le traitement des déchets étudiés.
\end{abstract}

\section{Introduction}

L'industrialisation, l'urbanisation et l'accroissement de la consommation au Maroc entraînent des problèmes de gestion et de traitement des déchets solides (ménagers, industriels et hospitaliers) et la prolifération de décharges non contrôlées (El Khamlichi et al., 1997).

Le Maroc produit environ 7,5 millions de tonnes de déchets solides par an dont 6,5 millions de tonnes de déchets ménagers, soit une moyenne par habitant de 0,75 gg par jour (MATEE, 2004). Prés de $70 \%$ de ces déchets sont concentrés dans le milieu urbain.

Les déchets ménagers produits par la province d'El Jadida sont riches en matières fermentescibles avec un pourcentage de l'ordre de $70-80 \%$ de poids sec et un taux d'humidité élevé de l'ordre de 80 \% de matière fraîche (Tazi, 200 I).

Les communes côtières de la ville d'El Jadida produisent des quantités élevées de déchets de poulets (28 900 tonnes/an) qui se caractérisent par une teneur élevée en azote et une humidité faible de 30 \% de matière fraîche (Tazi, 200 I). Ce dernier déchet est souvent utilisé en agriculture sans aucun traitement préalable. Les caractéristiques physico-chimiques de ces deux types de déchets et la vocation agricole de la région justifient une réflexion sur la faisabilité du compostage comme voie de stabilisation de ces déchets.

Dans ce travail, deux types de composts ont été obtenus lors du compostage du mélange de déchets ménagers et de déchets de poulets. La technique de biodégradation aérobie choisie est le compostage en réacteur. Le compostage en réacteur permet le contrôle des paramètres physico-chimiques tels que la température, l'aération, I'humidité, etc. II permet une optimisation du processus de biodégradation dans un volume réduit, et aussi d'éviter les odeurs causées par la production de composés volatiles très odorants lors de la phase thermophile (DeVleeschauwer et al., 1981, De Bertoldi, 1993).

Les conditions d'aération et le choix des critères de constitution des mélanges ont permis le développement d'une activité microbiologique intense durant la phase de fermentation dans les réacteurs et une bonne maturation des composts obtenus dans les sacs perforés. L'évolution de la maturité des composts a été suivie à travers un test phytotoxique et des analyses chimiques (C/N et les formes d'azote minéral).

\section{Matériel et méthodes}

\section{II.I Compostage}

\section{II.I.I Substrats utilisés}

Les déchets ménagers sont collectés dans la décharge publique de la ville d'El Jadida. Avant la mise en route des essais de compostage, les déchets ménagers ont subi un prétraitement permettant d'éliminer les éléments dont la taille est supérieure à $5 \mathrm{~cm}$, tels que verres, métaux, papiers et plastiques. 
Ces éléments peuvent nuire au processus de compostage (apport d'un taux élevé de carbone organique et de métaux lourds), ainsi qu'à l'aspect esthétique du produit final (plastiques et verres). Par exemple, l'expérience des unités de traitement des ordures ménagères (UTOM) de la ville de Rabat et de la ville d'Agadir (Maroc) a prouvé que la granulométrie grossière des composts produits diminue leur valorisation agricole (Hafid et al., 200l). Après le tri, les matières organiques restantes ont été analysées (tableau I).

Les déchets de poulets proviennent d'une unité d'élevage se trouvant dans la commune de Tnine Chtouka (tableaul). L'activité microbienne dépend en premier lieu de la manière dont est constitué le mélange. Les déchets ménagers présentent une humidité élevée de l'ordre de $86 \%$ de matière fraîche et un rapport C/N de l'ordre de 34,9 (tableau I). Le déchet de poulet a une humidité faible (30\%) et un rapport C/N faible ( I0) (tableau I). L'humidité optimale de compostage se situe entre 60 et $70 \%$ (Finstein et Miller, 1985). Le déchet de poulet est utilisé comme matrice absorbante et comme agent pour ajuster le rapport $\mathrm{C} / \mathrm{N}$.

Deux composts ont été préparés en mélangeant la matière fraîche des DM et DP dans les proportions 3/2 (compost $\mathrm{Cl}$ ) et 2/3 (compost C2). Les deux mélanges ont subi un broyage avant la mise en compostage. La taille de la particule du mélange constitué des DM et DP est de $0,2 \mathrm{~cm}$.

Tableau 1 : Caractérisation physico-chimique des déchets ménagers et des déchets de poulets bruts utilisés pour le compostage.

\begin{tabular}{|c|c|c|}
\hline Paramètre & Déchets ménagers & Déchets de poulets \\
\hline Humidité (\%) & 86,4 & 31,3 \\
pH & 5,1 & 8,3 \\
Conductivité électrique & 7,5 & 14,5 \\
(dS/cm) & 85,7 & 72,5 \\
Matière volatile (\% de MS) & 48,6 & 31 \\
Carbone organique (\% de & & \\
MS) & 1,4 & 3,1 \\
Azote total Kjeldahl (\% MS) & 34,9 & 10 \\
C/N & \\
\hline
\end{tabular}

Les caractéristiques du mélange $\mathrm{Cl}$ sont :

— matière fraîche : $\mathrm{pH} 8,2$ et humidité $60 \%$.

— matière sèche : COT 40,I \% ; NTK 2,6 \% ; C/N I5,4 et MV $74,7 \%$

Les caractéristiques du mélange C2 sont :

— matière fraîche : pH 7,9 et humidité $60 \%$.

— matière sèche : COT 37,2 \%; NTK 2,8 \% ; C/N I3, I et MV $70,7 \%$.

\section{II.I.2 Dispositif expérimental}

Chacun des deux mélanges de déchets organiques est introduit dans un réacteur conçu en $P V C$ de $20 \mathrm{~cm}$ de diamètre et de $40 \mathrm{~cm}$ de longueur. La quantité de mélange employé est en moyenne de $5 \mathrm{~kg}$ de MF. L'aération forcée des substrats est assurée par un compresseur d'air utilisant un débit constant d'une vitesse de $8 \mathrm{l} / \mathrm{min}$. La température dans les réacteurs est enregistrée en continu à l'aide d'un thermomètre placé au centre de la masse à composter. L'air sortant du réacteur est saturé en vapeur d'eau qui est piégée par le gel de silice.
La teneur en $\mathrm{CO}_{2}$ (mg/g MS) qui se dégage durant le processus de compostage est déterminée par piégeage de ce composé dans un tube en verre contenant de l'ascarite (hydroxyde de sodium sur support).

On effectue un brassage intermittent pendant le $6^{e}$ jour, assurant l'homogénéisation, l'aération des lacunes du substrat et la régénération des populations microbiennes. Le brassage permet de corriger les tendances au tassement et à la diminution de la porosité des substrats. Un substrat qui a tendance à se tasser, à colmater les vides lacunaires, ne pourra pas évoluer dans les meilleures conditions. Le taux d'oxygène lacunaire défini comme le pourcentage d'oxygène dans l'air des « vides » joue un rôle primordial dans le processus de compostage aérobie des déchets solides (Mustin, 1987).

Le traitement des déchets se déroule en deux phases :

- une phase de fermentation chaude qui dure 15 jours ;

- une phase de maturation des composts dans des sacs perforés qui dure 231 jours.

Les réacteurs sont arrêtés après 15 jours. La maturation des composts se poursuit dans des sacs perforés, qui permettent une aération lente à une température comprise entre 20 et $25^{\circ} \mathrm{C}$.

Des échantillons uniformes et représentatifs d'environ $50 \mathrm{~g}$ de substrat sont prélevés avant et après la phase de fermentation $(0 ; 15$ jours) et à différentes phases de maturation. Ces phases de maturation correspondent à des âges de composts de 40 ; 71 ; $105 ; 130$; 173 ; 207 et 246 jours. Les substrats prélevés subissent des analyses physico-chimiques. La présence de la phytotoxicité dans les échantillons prélevés est évaluée par le test de germination sur le cresson.

\section{II.2 Méthodes d'analyses}

Durant la phase thermophile, la quantité de $\mathrm{CO}_{2}$ dégagé est mesurée par simple pesée journalière des tubes absorbeurs (contenant de l'ascarite). L'activité biologique est contrôlée à travers le suivi du changement de la coloration (noire au départ) dans le tube d'ascarite. Afin d'éviter des pertes de $\mathrm{CO}_{2}$, l'ascarite est renouvelée lorsque la coloration (blanche) atteint les deux tiers du tube.

La température est mesurée chaque jour par un thermomètre placé au centre de la masse à composter dans chaque réacteur.

Le pH, la conductivité électrique dans l'extrait soluble à l'eau de la matière fraîche $1: 10(M / V)$ ainsi que la matière volatile sont déterminés selon la méthode (DI.VA.P.R.A. 1998). Une masse connue $\mathrm{MI}$ de substrat est séchée à $105{ }^{\circ} \mathrm{C}$ puis calcinée au four à $550{ }^{\circ} \mathrm{C}$, pendant 24 h. La masse M2 obtenue est alors pesée.

Le pourcentage de matière volatile est donnée par :

$$
\% M V=(M I-M 2) / M I \times 100
$$

Le carbone organique, l'azote total et l'azote minéral sont déterminés selon les normes AFNOR (1987).

Carbone organique : La matière organique est oxydée par un excès de dichromate de potassium ( $\mathrm{IN}$ ) en milieu acide. Le titrage en retour de l'excès de dichromate de potassium se fait par le sel de Mohr. 
Sur 0,05 g de matière sèche sont ajoutés $20 \mathrm{ml}$ de dichromate de potassium $(\mathrm{IN})$ et $15 \mathrm{ml}$ de $\mathrm{H}_{2} \mathrm{SO}_{4}(36 \mathrm{~N})$. L'échantillon est porté à ébullition à reflux pendant $5 \mathrm{mn}$. Après refroidissement, l'échantillon est complété à $200 \mathrm{ml}$ avec de l'eau distillée. Sur $25 \mathrm{ml}$ prélevé, on ajoute $5 \mathrm{ml}$ d'acide orthophosphorique $\left(\mathrm{H}_{3} \mathrm{PO}_{4}\right)$ et quelques gouttes de diphénylamine. L'excès de dichromate est dosé en retour par une solution de sel de Mohr (IN), jusqu'à virage du violet au vert.

Azote total : L'échantillon est minéralisé en milieu acide concentré et à chaud en présence d'un catalyseur. Ceci permet de transformer l'azote organique en azote ammoniacal. Ce dernier est distillé en milieu basique et dosé après avoir été piégé par l'acide borique.

Azote minéral : L'azote ammoniacal $\left(\mathrm{N}-\mathrm{NH}_{4}{ }^{+}\right)$et les nitrates $\left(\mathrm{N}-\mathrm{NO}_{3}{ }^{-}\right)$sont extraits au $\mathrm{CaCl}_{2}$ à un rapport $\mathrm{M} / \mathrm{N}=20 / 100$ après agitation pendant une heure. Après centrifugation, la détermination de $\mathrm{N}_{-} \mathrm{NH}_{4}{ }^{+}$est effectuée par distillation d'une prise d'essai de $25 \mathrm{ml}$ en présence de 0,25 g de MgO. Le distillat est récupéré dans $10 \mathrm{ml}$ d'acide borique $(2 \%)$ et quel-

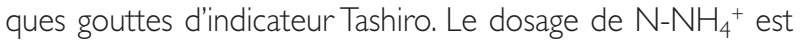
effectué par une solution de $\mathrm{H}_{2} \mathrm{SO}_{4}(0,2 \mathrm{~N})$. Sur la même prise d'essai $(25 \mathrm{ml})$ de l'extrait, est poursuivie et de la même manière une seconde distillation, après ajout de 0,25 g d'alliage de Devadra. Le dosage de $\mathrm{N}-\mathrm{NO}_{3}$ - est effectué de la même manière que celui de $\mathrm{N}-\mathrm{NH}_{4}{ }^{+}$.

\section{II.3 Test de phytotoxicité}

La phytotoxicité est un des derniers phénomènes qui permet de distinguer facilement un compost mûr d'un compost immature (Mustin, 1987 et Epstein, 1997).

Pour évaluer la maturité des composts obtenus, nous avons déterminé l'effet de l'extrait aqueux du compost sur la germination des graines de cresson (Lepidium sativum). Le test de cresson est un test rapide qui donne une réponse en 5 jours, en raison de la sensibilité de cette espèce (Zucconi, 1983). La maturité est évaluée suivant le pourcentage de germination.

L'extrait du compost est préparé selon la méthode (DI.VA.P.R.A, 1998). Une proportion I/5,7 (M/V) (compost (g de matière sèche) / eau distillée $(\mathrm{ml})$ ) est préparée pour chaque test de compost. Le compost/eau distillée est laissé en contact pendant 2 heures. Ce mélange est ensuite centrifugé à 600 rpm pendant 15 minutes. Le surnageant est filtré 2 fois sur papier filtre de diamètre 0,8 $\mu \mathrm{m}$. Deux dilutions sont préparées à partir du filtrat final pour avoir une concentration de 50 et $75 \%$ de l'extrait. Les graines sont trempées dans l'eau distillée pendant I h et mises à germer dans des boîtes de Pétri contenant du papier filtre ( 10 graines par boîte constitue une répétition). 10 boîtes sont imbibées chacune avec 5 $\mathrm{ml}$ de l'extrait aqueux du compost (5 boittes pour chaque dilution). Les cinq boîtes témoins reçoivent $5 \mathrm{ml}$ d'eau distillée. La germination a lieu à l'obscurité à $27^{\circ} \mathrm{C}$. Le nombre de graines germées par boîte est déterminé et la longueur de la racine est mesurée après 2 jours.
L'indice de germination IG (Zucconi, 1983) est défini comme étant le rapport :

$\mathrm{IG}$ = Nombre moyen de graines germées $\times$ Longueur de la racine / (Nombre moyen de graines germées $\times$ Longueur de la racine) (du témoin)

On désigne par IG 50 et IG 75 les indices de germination des graines irriguées avec l'extrait aqueux du compost correspondant respectivement aux dilutions 50 et $75 \%$. On définit l'indice de germination calculé pour nos essais comme la moyenne :

$$
I G=(I G 50+1 G 75) / 2
$$

\section{II.4 Traitements statistiques}

La réponse aux différents traitements utilisés dans le test de phytotoxicité est testée par l'analyse de la variance. La comparaison des moyennes est effectuée par la méthode de Newman-Keuls (Gouet et Philippeau, 1989).

\section{Résultats et discussions}

\section{III.I Activité microbienne pendant la phase thermophile}

Une des caractéristiques principales du compostage est la production de la chaleur due aux réactions exothermiques d'oxydation. Cette production de la chaleur représente une mesure indirecte de l'activité microbiologique. La température du substrat ne dépend pas seulement de la chaleur produite, mais également d'autres facteurs tels que la vitesse du flux d'air, son humidité, les échanges thermiques et la composition et la dimension particulaire du substrat (Chad et al., 200 I).

Figure 1 : Evolution de la production de $\mathrm{CO} 2$ et de la température au cours du temps pendant la phase thermophile des mélanges $\mathrm{Cl}$ et $\mathrm{C2}$

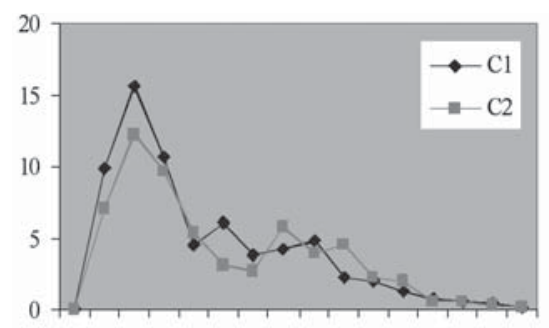

durée de fermentation chaude (j)

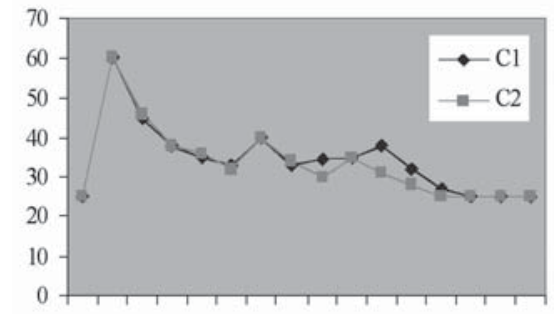

durée de fermentation chaude (j) 
L'activité biologique a été déterminée par la mesure journalière de la température des substrats dans les réacteurs et la production du $\mathrm{CO}_{2}$. Les courbes d'évolution de ces deux paramètres en fonction du temps sont présentées dans la figure I. Globalement, les variations de température et la production de $\mathrm{CO}_{2}$ montrent qu'il y a une grande similitude dans le déroulement des fermentations en réacteur pour les deux mélanges.

Dés le premier jour de compostage, la température et la production de $\mathrm{CO}_{2}$ augmentent. Trois phases successives d'activité peuvent être observées au cours de la stabilisation :

- la première phase est caractérisée par une croissance rapide des populations microbiennes mésophiles. La température passe de 25 à $60^{\circ} \mathrm{C}$ en une journée. La production de $\mathrm{CO}_{2}$ atteint au deuxième jour son maximum de 15,58 g pour $\mathrm{Cl}$ et de 12,2 g pour C2. Cette phase correspond à l'hydrolyse des composés facilement biodégradables tels que la cellulose et l'hémicellulose, et la production des acides gras volatiles (Mc Kinley et Vestal, 1985 et Domfizel et al., 1996) ;

- la deuxième phase montre un ralentissement de l'activité biologique. La température tend à se stabiliser vers 33 et $32{ }^{\circ} \mathrm{C}$ respectivement pour $\mathrm{Cl}$ et $\mathrm{C} 2$ pendant quatre jours. La production de $\mathrm{CO}_{2}$ baisse et atteint 3,83 et 2,62 g/j respectivement pour $\mathrm{Cl}$ et $\mathrm{C} 2$;

- la troisième phase est caractérisée par une stabilisation de la température des deux mélanges à $40{ }^{\circ} \mathrm{C}$ et une augmentation de la production de $\mathrm{CO}_{2}$ de l'ordre de 4,72 g/j pour $\mathrm{Cl}$ et 4,56 g/j pour C2. Cette augmentation est due à la bonne diffusion de l'air dans les réacteurs, causée par un brassage manuel réalisé le sixième jour et entraînant une activité microbienne.

Après la troisième phase, apparait une phase d'activité biologique lente, en réaction à l'épuisement des substances nutritives du substrat. Durant cette dernière phase, la température et la production de $\mathrm{CO}_{2}$ baissent progressivement pour atteindre respectivement $25^{\circ} \mathrm{C}$ et $0,38 \mathrm{~g} / \mathrm{j}$ pour $\mathrm{Cl}$ et $25^{\circ} \mathrm{C}$ et $0,14 \mathrm{~g} / \mathrm{j}$ pour $\mathrm{C} 2$. La température $25^{\circ} \mathrm{C}$ est notée après 12 jours pour $\mathrm{Cl}$ et 13 jours pour $\mathrm{C} 2$.

L'absence de toute augmentation de température après le

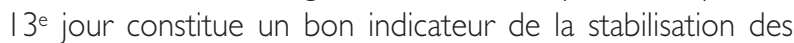
composts (Mustin, 1987). Les températures atteintes dans les mélanges $\mathrm{Cl}$ et $\mathrm{C} 2$ permettent une hygiénisation et une biodégradation maximale des substrats (Miller, 1995 et Tazi, 200I). Le compost brut obtenu est stabilisé et il fermente encore lentement : c'est la phase de maturation.

\section{III.2 Evaluation de la maturité du compost}

\section{III.2.I Tests physico-chimiques}

\section{- $\mathrm{pH}$ et CE}

D'après la figure 2, le pH de la solution aqueuse du compost a subi une augmentation à partir de la phase thermophile. La solution du compost est passée d'un $\mathrm{pH}$ légèrement alcalin $(8,2$ et 7,9 respectivement pour $\mathrm{Cl}$ et $\mathrm{C2}$ ) à un pH alcalin (9 et 8,9 respectivement pour $\mathrm{Cl}$ et C2) au 40 jour. Pendant la phase thermophile, l'alcalinisation du milieu est liée à l'ammoniac (base) produit par l'hydrolyse bactérienne de l'azote protéique et organique. Au cours de la maturation, le $\mathrm{pH}$ reste basique puis diminue progressivement en fonction du temps pour atteindre la neutralisation. La stabilité du $\mathrm{pH}$ est due aux réactions lentes de maturation et au pouvoir tampon de l'humus (Fauci et al., 1999).

\section{Figure 2 : Evolution de la conductivité électrique à $25^{\circ} \mathrm{C}$ et du $\mathrm{pH}$} en fonction du temps de compostage des mélanges $\mathrm{C} 1$ et $\mathrm{C} 2$.
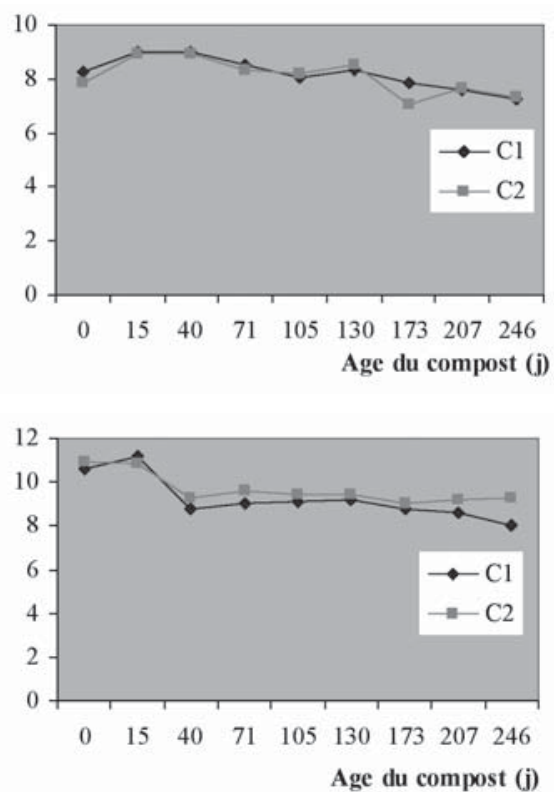

La figure 2 montre que la conductivité électrique est élevée au début du compostage, 10,6 et $10,9 \mathrm{dS} / \mathrm{m}$ pour $\mathrm{Cl}$ et $\mathrm{C} 2$ respectivement, puis diminue en fonction du temps de compostage pour atteindre 8 et 9,3 dS/m respectivement pour $\mathrm{Cl}$ et $\mathrm{C} 2$. Ces valeurs restent cependant élevées pour le compost. Elles sont dues essentiellement à la concentration élevée des nutriments (l'azote, le calcium, le magnésium, le potassium, le sodium et le phosphore).

\section{- Azote minéral}

Au cours du processus de compostage, la quantité d'azote ammoniacal extractible décroît progressivement en fonction de l'âge du compost alors que la quantité de nitrate augmente (figure 3). Au début du compostage, la teneur en ammonium est de 0,21 \% pour $\mathrm{Cl}$ et de $0,17 \%$ pour $\mathrm{C} 2$, celle en nitrates étant faible. A la fin de la maturation, la quantité d'ammonium devient $0,05 \%$ pour $\mathrm{Cl}$ et $0,07 \%$ pour $\mathrm{C} 2$ alors que celle des nitrates est de $0,1 \%$ pour $\mathrm{Cl}$ et de $0,09 \%$ pour C2.

Le passage de l'azote ammoniacal à l'azote nitrique s'effectue par la minéralisation des composés azotés complexes en ammoniac et en acides aminés. L'ammonium peut être soit utilisé directement dans le métabolisme microbien, soit être oxydé en nitrates et nitrites, par les organismes fixants d'azote. Dans ce travail, nous avons utilisé l'ammonium et les nitrates, d'une manière combinée, comme indicateur de la maturité du compost testé. Un compost mûr étant pauvre en ammonium et riche en nitrate (Fauci et al., 1999). 
Figure 3 : Evolution des formes N-NH4+ et N-NO3- durant le compostage des mélanges $\mathrm{Cl}$ et $\mathrm{C2}$.
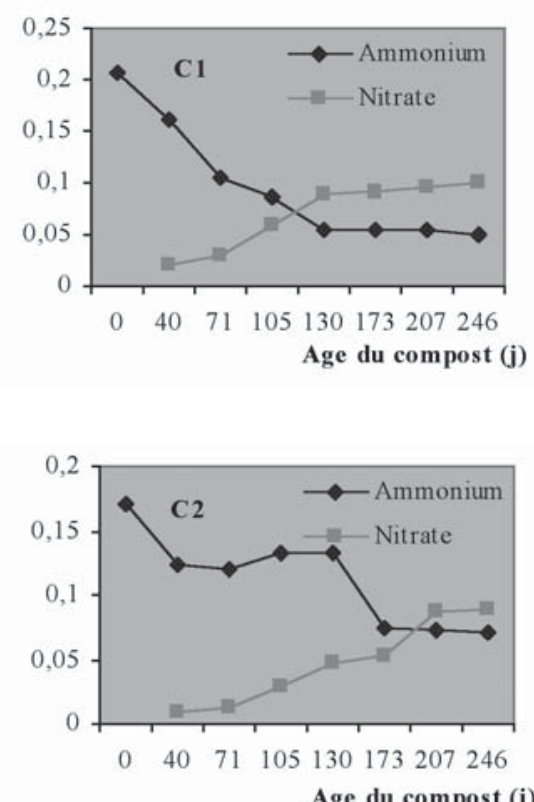

\section{- Rapport $\mathbf{C / N}$}

Le rapport $\mathrm{C} / \mathrm{N}$ suit la même évolution pour les deux mélanges (figure 4). II est de 15,4 et |3, I respectivement pour $\mathrm{Cl}$ et $\mathrm{C2}$ au début de compostage.

Figure 4 : Evolution du rapport $\mathrm{C} / \mathrm{N}$ des composts $\mathrm{Cl}$ et $\mathrm{C} 2$ durant le compostage.

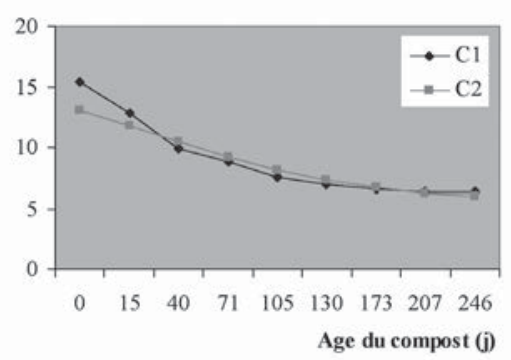

Durant la phase de maturation, le rapport $\mathrm{C} / \mathrm{N}$ subit une diminution. Après 246 jours de compostage, il tend vers 6,3 pour $\mathrm{Cl}$ et 6 pour $\mathrm{C} 2$. L'évolution du rapport $\mathrm{C} / \mathrm{N}$ est en relation directe avec la biodégradation de la matière organique qui se traduit à la fois par l'élimination du carbone sous forme de $\mathrm{CO}_{2}$ et par la concentration apparente des éléments minéraux ( $N, P, K \ldots)$. D'un autre côté, il y a des pertes d'azote, sous forme d'ammoniac, au cours de la phase thermophile.
Ces pertes ont tendance à atténuer la baisse du rapport $\mathrm{C} / \mathrm{N}$. Des résultats similaires ont été rapportés par d'autres auteurs (Hafidi, 1996 et Bousselhaj et al., 1996).

Le rapport $\mathrm{C} / \mathrm{N}$ est un autre indicateur de la maturité du compost (Lhadi et al., 2004 et 2006, Aylaj, 2002, Tazi, 200I, Ozores-Hampton et al., 1998 et Mathur et al., 1993).

\section{III.2.2 Test de phytotoxicité}

Les résultats de l'analyse de variance ont montré des différences hautement significatives entre les différents âges de compost, aussi bien pour l'indice de germination que pour la longueur de la racine. L'interaction compost-âge et la différence entre les deux composts sont hautement significatives pour l'indice de germination.

En moyenne, l'indice de germination (IG) et la longueur de la racine $(L R)$ sont nuls pendant la phase thermophile pour les deux traitements ( $\mathrm{Cl}$ et $\mathrm{C} 2$ ) (figure 5 ). Après cette phase, ces deux paramètres augmentent progressivement avec l'augmentation de l'âge du compost, sans présenter de variation notable entre $\mathrm{Cl}$ et C2 (figure 5).

Figure 5 : Evolution de l'indice de germination des graines ef la longueur de la racine du cresson (Lepidium sativum ) en fonction de I'âge du compost.
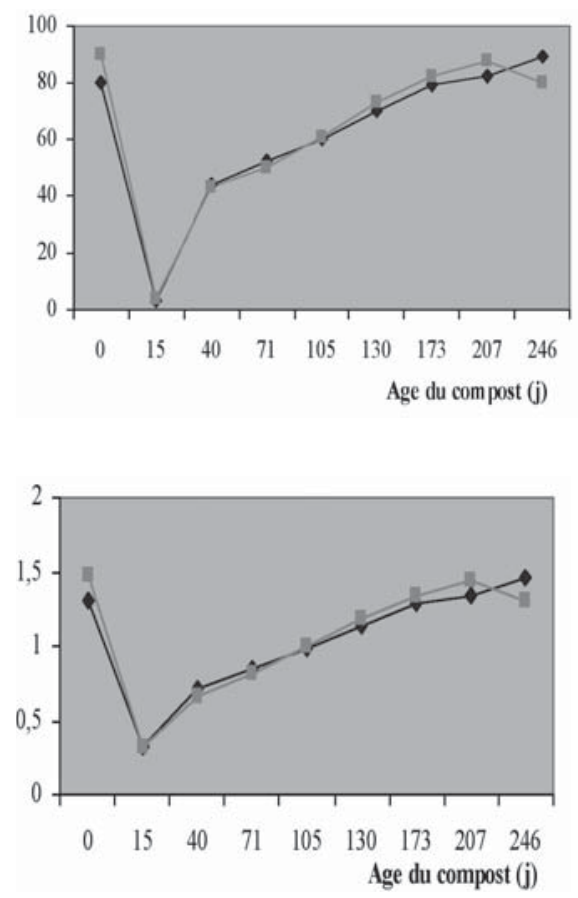

Lorsque la phase thermophile s'achève, le compost brut obtenu est stabilisé. II entre dans une phase où les réactions de production de l'humus (humification) sont prédominantes. Le compost brut stabilisé, au stade de la préhumification, est de plus phytotoxique. Utilisé directement pour la germination des graines de cresson, il les inhibe en libérant dans le milieu de germination de l'ammoniac et des composés organiques transitoires en quantités supérieures aux seuils de toxicité des graines de cresson. 
La phase thermophile correspond au stade de dégradation intense de la majorité de la matière organique, aboutissant ainsi à la production de substances toxiques tels que l'ammoniac, l'acide acétique, l'acide propionique (Kirchmann et Widén, 1994). La présence de ces acides dans l'extrait du compost inhibe la germination des graines de cresson (Barberis et Nappi, 1996 et Ozores-Hampton et al., 1999). La concentration élevée de l'ammonium (figure 3) dans les composts $\mathrm{Cl}$ et $\mathrm{C} 2$ contribue indubitablement à la phytotoxicité (Grebus et al., 1994).

L'inhibition de la germination du cresson est surtout due à l'acide acétique. Ce composé peut inhiber complètement la germination des graines de cresson à partir d'une concentration de 300 mg/kg (DeVleeschauwer et al., 198I). La réduction de la germination des graines par l'acide acétique du compost a été rapportée pour le cresson (Lepidium sativum), les oignons (Allium cepa $L$ ), les choux (Brassica oleracea $L$ ), le chou-fleur (Brassica oleracea L), la laitue (Lactuca sativa L), la tomate (Lycopersicon esculentum mill) et le maïs (Zea mays $L$ ) (Keeling et al., 1994 et Ozores-Hampton et al., 1999).

Pendant les dernières phases de compostage, le rapport C/N diminue, les réactions deviennent plus lentes. De l'ammoniac est perdu par volatilisation et l'azote est utilisé par les microorganismes pour réaliser la biosynthèse des matières humiques. La phytotoxicité diminue puis disparaît complètement à la fin de la phase de maturation. Ainsi, l'indice de germination pour le cresson atteint 89 et $76 \%$ respectivement pour $\mathrm{Cl}$ et $\mathrm{C} 2$. Ceci indique que le compost produit est stable et mature.

Le test de Newman-Keuls a permis de classer l'indice de germination par rapport à l'âge du compost (exprimé en jours de compostage) en sept groupes homogènes (0,207 et 246) $>173>130>105>71>40>15$ et par rapport au type de compost, en deux groupes homogènes $\mathrm{C} 2>\mathrm{Cl}$. La longueur de la racine a été classée par rapport à l'âge du compost (exprimé en jours de compostage) en six groupes homogènes $(0,207$ et 246$)>173>130>105>(7 \mid$ et 40$)$ $>15$.

\section{Conclusions}

Les mélanges $\mathrm{Cl}$ et $\mathrm{C} 2$ ont atteint la température de $60^{\circ} \mathrm{C}$ durant la phase thermophile. Le taux de $\mathrm{CO}_{2}$ produit a atteint 15,6 g/j dans $\mathrm{Cl}$ et $12,2 \mathrm{~g} / \mathrm{j}$ dans $\mathrm{C} 2$. A la fin de la maturation, la quantité d'ammonium atteint $0,05 \%$ pour $\mathrm{Cl}$ et $0,07 \%$ pour $\mathrm{C} 2$. Après 246 jours de compostage, le rapport C/N a convergé vers 12,6 pour $\mathrm{Cl}$ et 12 pour $\mathrm{C} 2$.

Le test de phytotoxicité a montré que l'indice de germination du cresson est nul durant la phase de stabilisation pour les deux composts. L'indice de germination $40 \%$ est obtenu à partir de 40 jours de compostage pour les deux types de composts. A 105 jours de compostage l'IG dépasse $60 \%$ pour les deux composts.
L'utilisation combinée des paramètres physico-chimiques et du test phytotoxique sur le cresson a permis de confirmer la stabilité et la maturité des composts $\mathrm{Cl}$ et $\mathrm{C}$ 2. Les essais biologiques constituent un complément important à la caractérisation physico-chimique de la qualité du compost.

Les composts obtenus sont de bonne qualité et le compostage peut donc être une bonne voie pour le traitement des déchets étudiés.

\section{Références bibliographiques}

AFNOR, (1987). Qualité des sols et méthodes d'analyse. I ère édition, $133 \mathrm{p}$.

Aylaj M. (2002). Effet de la salinité sur la betterave à sucre, valorisation agricole du compost et son effet sur l'amélioration de la tolérance de la betterave à sucre (Beta vulgaris $L$ ) : Approche physiologique et environnementale. Thèse de Doctorat, Faculté des Sciences, El Jadida, Maroc. pp : I 46. Bernal M.P., Roig P., Sanchez-Monodero A., Paredas C. et Garcia D., (1996). Nitrogen In : Composting Science and Utilization, De Bertoldi M ., Sequi P., Lemmes B. et Papi T., (eds), European commission. International Symposium. Blackie Academic and Professional, 1074- 1077.

Bousselhaj K., Ouazzani N., Hafidi M., Morel J.L. et Revel J.C., (1996). Composting effect on agronomic value of two Sewage Sludge. International Symposium on Environmental Impact and Risk Assessment, Mohammedia, October, 9- I I th Maroc.

Chad W., Higgings et Walker L.P., (200 I). Validation of a new model for aerobic organic solids decomposition : simulation with substrate specific kinetics. Process Biochemistry, 36 : 875 884.

Cooney C.L., Wang D.I.C. et Mateles R.I., (1969). Measurement of heat evolution and correlation with oxygen consumption during microbial growth. Biotechnology and Bioengineering. 9, $3: 269-281$.

De Bertoldi M., (1993). Compost quality and standard specifications. In : Science and Engineering of Composting. Design, Environmental, Microbiological and Utilization Aspects. Ed. H.A.I Hointik and H.M. Keener. The Okio State University, 523-535.

DeVleeschauwer D. O., Verdonock P. et VanEssche P., (198I). Phytotoxicity of refuses compost. Biocycle. 22, I :44-46. DI. VA. PR. A. et I.P.L.A., (1992). Umidita totale, Umidita residua, Sostanze volatilie Ceneri conducibilitae salinita, II saggio di fitotossicita. In : DI.VA.P.R.A. et I.P.L.A. (eds). Metodi di analisi dei Compost. Collana Ambiente, Assessorato all' Ambiente, Regione Piemonte, pp 70-73.

DI. VA. PR. A., I.P.L.A. et A.R.P.A., (1998). Metodi di Analisi dei compost. Determination chiniche, Fixiche, Biologiche e Microbiologiche. Analisi Merceologica die fifiuti. In Di. V.P.R.A, I.P.L.A et A.R.P.A eds .Metodi di analisi dei compost, collana Ambiante, Torino, pp 85-86.

Domfizel M., Valentin N. et Massiani P., (1996). Monitoring of organic matter during composting. The Science of composting. Part II Ed, Debertoldi M. Sequi P., Lemmes B. et Papi T. : European Commission, International Symposium. I 42. 
El Khamlichi M.A. et al "(1997). Etude d'impact de la décharge publique d'Akrach sur la qualité des ressources en eau. Revue marocaine de génie civil N 68, 17-31.

Epstein E., (1997). The science of composting. Technonic Publishing Company Inc. Lancaster, PE, USA.

Fauci M.F., Berdicek F., Caldwell D. and Finch R, (1999). End product quality and agronomic performance of compost. Compost Science and Utilization, vol 7, n (2), 17-29.

Finstein M.S. et Miller F.C., (1985). Principles of composting lead to maximization of decomposition rate, control and cost effectiveness. In : Composting of Agricultural and other Wastes. Ed : Gasser I.K.R., Elsevier Applied Science, I3-26.

Genevini P.L., Adani F. et Villa C., (1997). Rice hull degradation byco-composting with dairy cattle slurry. Soil Science and Plant Nutrition. 43,1 : : 135- 147.

Gouet J.P. et Philipeau G., (1989). Comment interpréter les résultats d'une analyse de variance. Institut technique des Céréales et des Forages. Document STAT. ITCF.

Grebus M.E., Watson M.E. and Hoitink H.A.J., (1994). Biological, chemical and physical properties of composted yard trimmings as indicators of maturity and plant disease suppression. Compost Science and Utilization, vol: 2, I, pp :57-71.

Hafid N., El Hadek M. et Lguirati A., (200 I). Caractérisation du compost urbain et des dépôts de la décharge dans la ville d'Agadir. Annale de chimie Science et Matériaux, 26, pp : $44|-45|$.

Hafidi M., (1996). Contribution à la valorisation des composts par addition de phosphates naturels marocains. Thèse de doctorat d'Etat. Univ. Cadi Ayyad, Fac. Sci. Semlalia, Marrakech. I 12 p.

Keeling A.A., Paton I.K. et Mullet J.A., (1994). Germination and growth of plants in media containing unstable refuse derived compost. Soil Biol. Biochem., 26, 767-772.

Kirchmann H. And Widén P., (1994). Fatty acid formation during composting of separately collected organic household wastes. Compost science and utilization vol : 2, I, pp : 17-19.

Levitt J., (1980). Water, radiation, salt and other stresses, in responses of plants to environmental stress. Academic press, London, 607 pages.

Lhadi E.K., Tazi H., Aylaj M., Tambone F. and Adani F., (2004). Co-composting separated MSW and poultry manure in Morocco. Compost Science and Utilization vol. I2, N 2, I37- I 44.
Lhadi E.K., Tazi H., Aylaj M., Genevini P.L. and Adani F., (2006). Organic matter evolution during co-composting the organic fraction of municipal waste and poultry manure. Bioressource Technology vol. 97, 21 17-2123.

M.AT.E.E., (2004). Pour une gestion intégrée des déchets solides ménagers au Maroc. Ministère de l'aménagement du territoire, de l'eau et de l'environnement. Conférance à Rabat (Maroc), 3 mars.

Mathur S.L., Owen G., Dinel H. et Schnitzer M., (1993). Determination of compost biomaturity. Literature review. Biological Agriculture and Horticulture, 10, 65-85.

McKinley V.L. et Vestal R.V., (1985). Physical and chemical correlates of microbiol activity and biomass in composting municipal sewage sludge. Applied and Environmental Microbiology, 50 (90), I 395- 1403.

Mustin M., (1987). Le compost, gestion de la matère organique. Ed. François Dubux, Paris, 954 p.

Miller F.C., (1995). Heat evolution during composting of sewage sludge. In : Composting Science and Utilization. De bertoldi M., Sequi P., Lemmes B. et Papi T. (eds), European Commission. Iternational Symposium. Blackie Academic and Professional. I06-I I 5.

Ozores-Hampton M.P., Obreza T.A. and Hochmuth G., (1998). Composted municipal solid waste use on vegetable crops. Hort Technology. 8 : I0- 17.

Ozores-Hampton M.P., Vavrina C.S. et Obreza T.A., (1999). Yard trinming biosolids compost: Possible alterative to sphagnum peat moss in Tomato transplant production. Compost science and Utilization.,7 : 4, 42-49.

Shiralipour A. Mc Connell D.B. et Smith W.H., (1997). Phytotoxic effect of a short-chain fatty acid on seed germination and root length of Cucumis sativus CV Poinset. Compost Science and Utilization. 5 : 2. 47-52.

Tazi H., (200I). Déchets solides : Etude d'impact sur l'environnement (sol, eaux souterraines) et traitement par voie de compostage. Thèse de Doctorat Faculté des Sciences El Jadida, Maroc. pp : 224.

Zhar J., (1989). Quand progrès rime avec déchet. Enjeux, 7 : $33-42$.

Zucconi F., (1983). Processi di biostabilizzazione della Sostanza organica durante il compostaggio. Atti del Simposio Recupero biologico ed Utilizzazione agricola dei rifuiti urbanis. Napoli, I I- 44 octobre, p : 379-406.

\section{DÉCHETS SCIENCES \& TECHNIQUES, REVUE FRANCOPHONE D’ÉCOLOGIE INDUSTRIELLE SAP - 9, rue de l'Arbre Sec - 6928I LYON CEDEX 0I- Mèle : olivier.guichardaz@pro-environnement.com Service abonnement : SAP/DPE - Service abonnement - 9, rue de l'Arbre Sec - 6928I LYON CEDEX 0I \\ Tél. : 0472982669 - Fax : 0472982680}

$\mathrm{N}^{\circ}$ de commission paritaire : $0307 \mathrm{~T} 88295$ - N ISSN : 0753-3454. Dépôt légal : À parution - Imprimerie Louis Jean/Gap - Photocomposition SAP Principaux associés : DPE 